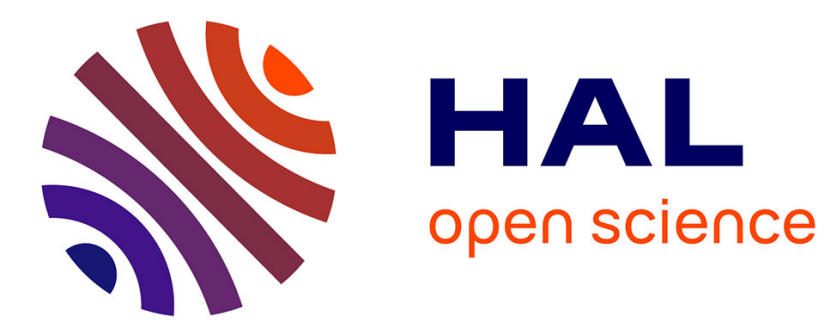

\title{
Computation of conjugate times in smooth optimal control: the COTCOT algorithm
}

\author{
Bernard Bonnard, Jean-Baptiste Caillau, Emmanuel Trélat
}

\section{To cite this version:}

Bernard Bonnard, Jean-Baptiste Caillau, Emmanuel Trélat. Computation of conjugate times in smooth optimal control: the COTCOT algorithm. 2005, 6 p. hal-00086460

\section{HAL Id: hal-00086460 https://hal.science/hal-00086460}

Submitted on 18 Jul 2006

HAL is a multi-disciplinary open access archive for the deposit and dissemination of scientific research documents, whether they are published or not. The documents may come from teaching and research institutions in France or abroad, or from public or private research centers.
L'archive ouverte pluridisciplinaire HAL, est destinée au dépôt et à la diffusion de documents scientifiques de niveau recherche, publiés ou non, émanant des établissements d'enseignement et de recherche français ou étrangers, des laboratoires publics ou privés. 


\title{
Computation of conjugate times in smooth optimal control: the COTCOT algorithm
}

\author{
Bernard Bonnard, Jean-Baptiste Caillau and Emmanuel Trélat
}

\begin{abstract}
Conjugate point type second order optimality conditions for extremals associated to smooth Hamiltonians are evaluated by means of a new algorithm. Two kinds of standard control problems fit in this setting: the so-called regular ones, and the minimum time singular single-input affine systems. Conjugate point theory is recalled in these two cases, and two applications are presented: the minimum time control of the Kepler and Euler equations.
\end{abstract}

\section{INTRODUCTION}

We consider a smooth Hamiltonian equation

$$
\dot{z}=\vec{H}(z)
$$

on the cotangent bundle of a smooth manifold $M$. Such an equation arises in the optimal control of systems with smooth control. Indeed, extremal trajectories are parameterized by Pontryagin maximum principle and satisify the standard Hamiltonian equation. In the two cases of regular systems, and singular single-input affine minimum time systems, the control is smooth and a Hamiltonian equation of the form (1) is derived. Moreover, second order conditions for (local) optimality of a given extremal, $z$, can be checked by computing a set of solutions to the variational system along the extremal:

$$
\delta \dot{z}=d \vec{H}(z(t)) \delta z .
$$

System (2) is called the Jacobi equation. This kind of second order conditions are known as conjugate point conditions [1], [2], [3]. An implementation of the relevant computations, including solving (1) and (2) is provided by the Matlab package cotcot [4]. More precisely, on the basis of a userprovided Hamiltonian, the second members of (1) and (2) are evaluated by automatic differentiation [5]. The numerical integration of the differential equations and the solution of the associated shooting problem are computed by standard Netlib codes interfaced with Matlab. We propose two applications of the algorithm in spaceflight dynamics: first to orbit transfer, then to attitude control.

To this end, we first recall in $\S I I$ and $\S I I I$ the conjugate point theory, respectively for regular control problems and minimum time singular single-input affine systems. Then, the minimum time control of the Kepler equation is presented in $\S I V$. The aim is to compute orbit transfers around the Earth

This work was supported by the French Space Agency under contract 02/CNES/0257/00-DPI 500

B. Bonnard is with the Institut de Mathématiques, Université de Bourgogne, F-21078 Dijon Bernard. Bonnardeu-bourgogne. fr

J.-B. Caillau is with ENSEEIHT-IRIT (UMR CNRS 5505), 2 rue Camichel, F-31071 Toulouse caillau@n7. fr

E. Trélat is with the AN-EDP Lab., Université de Paris-Sud, F-91405 Orsay emmanuel.trelat@math.u-psud.fr and to check optimality of the corresponding extremals. This is done in the regular multi-input case as well as in the singular single-input exceptional case. The second application is the attitude control of a spacecraft. A preliminary study of the Euler equations is achieved. The hyperbolic and exceptional singular cases of the single-input system are finally analyzed in $\S \mathrm{V}$. For a more detailed presentation of the topic, we refer readers to [6], [7].

\section{REGULAR CONTROL SYSTEMS}

Consider the control of the system

$$
\dot{x}=f(x, u), x(0)=x_{0}
$$

where $x$ belongs to a smooth manifold $M$ identified with $\mathbf{R}^{n}$, and where the cost to minimize is the functional

$$
C(x, u)=\int_{0}^{T} f^{0}(x, u) d t .
$$

The right hand side $f: \mathbf{R}^{n} \times \mathbf{R}^{n} \rightarrow \mathbf{R}^{n}$ is smooth and $u$ takes values in $\mathbf{R}^{m}$. Since the control domain is unbounded, every optimal control $u$ on $[0, T]$ is a singularity of the endpoint mapping $E_{x_{0}, t}: \mathrm{L}_{m}^{\infty}([0, t]) \rightarrow \mathbf{R}^{n}$ for $0<t \leq T$ where $E_{x_{0}, t}(u)=x\left(t, x_{0}, u\right)$ is the solution of (3): the Fréchet derivative at $u$ of the mapping is not surjective (its image has codimension at least one; see assumption (A2) hereafter). The resulting trajectory is the projection of an extremal $\left(x, p^{0}, p, u\right), p^{0}$ non-positive, solution of the maximum principle,

$$
\dot{x}=\frac{\partial H}{\partial p}, \dot{p}=-\frac{\partial H}{\partial x}
$$

and

$$
\frac{\partial H}{\partial u}=0
$$

where $H=p^{0} f^{0}(x, u)+\langle p, f(x, u)\rangle$ is the standard Hamiltonian, constant along the extremal, zero if the final time is free. The Hamiltonian is homogeneous in $\left(p^{0}, p\right)$ and we have two cases: the normal case where $p^{0}$ is not zero and normalized to $p^{0}=-1$, and the exceptional case otherwise, $p^{0}=0$. Without losing any generality, we may assume that the trajectory is one to one on $[0, T]$. We make the strong Legendre assumption,

(A1) The quadratic form $\partial^{2} H / \partial u^{2}$ is negative definite along the reference extremal.

Therefore, using the implicit function theorem, the extremal control can be locally defined as a smooth function $u_{r}$ of 
$z=(x, p)$, solution of $\partial H / \partial u=0$. This defines the regular Hamiltonian function

$$
H_{r}(x, p)=H\left(x, p^{0}, p, u_{r}(x, p)\right)
$$

and the reference extremal is a smooth solution of

$$
\dot{z}=\vec{H}_{r}(z) \text {. }
$$

Definition 2.1: Let $z=(x, p)$ be the reference extremal defined on $[0, T]$. The variational equation

$$
\delta \dot{z}=d \vec{H}_{r}(z(t)) \delta z
$$

is called the Jacobi equation. A Jacobi field is a non trivial solution $J$ of (5). It is said to be vertical at time $t$ if $\delta x(t)=$ $d \Pi(z(t)) \cdot J(t)=0$ where $\Pi:(x, p) \mapsto x$ is the standard projection.

In order to derive second order optimality conditions, we make the following additional generic assumptions on the reference extremal.

(A2) The singularity of the endpoint mapping $E_{x_{0}, t}$ at $u$ is of codimension one on each nonempty subinterval of $[0, T]$.

Let $\left(x\left(t, x_{0}, p_{0}\right), p\left(t, x_{0}, p_{0}\right)\right)$ denote the solution of (4) for the initial condition $\left(x_{0}, p_{0}\right)$.

Definition 2.2: For small enough nonnegative $t$, we define the exponential mapping by

$$
\exp _{x_{0}, t}\left(p_{0}\right)=x\left(t, x_{0}, p_{0}\right) \text {. }
$$

The domain of the exponential depends on whether we are in the exceptional case or not, and on whether the final time is fixed or not.

Definition 2.3: Let $z=(x, p)$ be the reference extremal defined on $[0, T]$. Under our assumptions, the time $0<$ $t_{c} \leq T$ is called conjugate if the mapping $\exp _{x_{0}, t_{c}}$ is not an immersion at $p_{0}$. The associated point $x\left(t_{c}\right)$ is said to be conjugate to $x_{0}$. We denote by $t_{1 c}$ the first conjugate time.

The fundamental result relating conjugate points to the optimality status of extremals in the regular case is the following [3], [1], [2].

Theorem 2.1: Under our assumptions, let $(x, p, u)$ be the reference regular extremal defined on $[0, T]$. It is locally optimal with respect to all trajectories with same extremities in the $\mathrm{L}^{\infty}$-topology ( $\mathscr{C}^{0}$-topology if the extremal is normal), up to the first conjugate time $t_{1 c}$. It is not locally minimizing in the $\mathrm{L}^{\infty}$-topology after $t_{1 c}$.

In the normal case with fixed final time, let $X$ be the $n$-dimensional fiber $T_{x_{0}}^{*} M$. Then, the exponential mapping is defined on an open subset of $X$ and, for a given $p_{0}$ in $X$, we denote by $L_{t}$ the transport of $L_{0}=T_{p_{0}} X$ by the variational equation along the extremal $z=(x, p)$ defined by $\left(x_{0}, p_{0}\right)$. Clearly, $L_{t}$ is a Lagrangian subspace of $T_{x(t)}^{*} M$ spanned by Jacobi fields vertical at $t=0$, and singularities of the exponential are detected by checking the rank of the projection of $L_{t}$ on the $x$-space. Therefore, $t_{c}$ is a conjugate point if and only if

$$
\operatorname{rank} d \Pi\left(z\left(t_{c}\right)\right) \cdot L_{t_{c}}<\operatorname{dim} X .
$$

In the three other cases, the test is still (6) but $X$ has to be restricted to suitable submanifolds of the fiber. In the normal case with free final time, the Hamiltonian is zero and $X$ is chosen according to

$$
X=\left\{p_{0} \in T_{x_{0}}^{*} M \mid H_{r}\left(x_{0}, p_{0}\right)=0\right\} .
$$

The exceptional case is treated similarly with, respectively,

$$
X=\mathbf{S}^{n-1} \subset T_{x_{0}}^{*} M
$$

if the final time is fixed, and

$$
X=\left\{p_{0} \in \mathbf{S}^{n-1} \subset T_{x_{0}}^{*} M \mid H_{r}\left(x_{0}, p_{0}\right)=0\right\}
$$

otherwise.

\section{SINGULAR SINGLE-INPUT AFFINE SYSTEMS, MINIMUM TIME}

We consider the minimum time control of a single-input affine system

$$
\dot{x}=F_{0}+u F_{1}
$$

where $F_{0}$ and $F_{1}$ are smooth vector fields on a manifold $M$ identified with $\mathbf{R}^{n}$, and $u$ is valued in $\mathbf{R}$. Optimal trajectories are singular, but we cannot apply the previous algorithms to check second order conditions because the strong Legendre condition is not satisfied anymore. Our aim is to apply the theoretical framework of [2] so as to get sufficient conditions, together with algorithms from [8]. We first introduce some generic conditions along the reference extremal. Let $x$ be the reference singular trajectory on $[0, T]$, and let $u$ be the associated control. First of all, it is convenient to apply a feedback transformation to normalize the control to $u \equiv 0$. We make indeed the following assumptions.

(A1) The reference trajectory is smooth and injective.

(A2) For every $t \in[0, T], \operatorname{Span}\left\{\operatorname{ad}^{k} F_{0} \cdot F_{1}(x(t)) \mid k=\right.$ $0, \ldots, n-2\}$ has codimension one.

As a result, this vector subspace is the Pontryagin cone $K(t)$ for positive $t$. The adjoint $p(t)$ is unique up to a constant and oriented with the convention $H \geq 0$ of the maximum principle.

(A3) Along the reference trajectory, the vector field $\operatorname{ad}^{2} F_{1} \cdot F_{0}$ does not belong to $\operatorname{Span}\left\{\operatorname{ad}^{k} F_{0} \cdot F_{1} \mid k=0, \ldots, n-2\right\}$.

This last condition implies that the reference singular extremal $z$ is a so-called order two extremal, solution of

$$
\dot{z}=\vec{H}_{s}(z)
$$

on $\left\{H_{1}=\left\{H_{0}, H_{1}\right\}=0\right\}$ with

$$
\begin{aligned}
& H_{s}=H_{0}+u_{s} H_{1} \\
& u_{s}=-\frac{\left\{H_{0},\left\{H_{0}, H_{1}\right\}\right\}}{\left\{H_{1},\left\{H_{0}, H_{1}\right\}\right\}} .
\end{aligned}
$$

Here before, the brackets stand for the standard Poisson bracket of smooth functions on the cotangent bundle. Our last assumption is as follows.

(A4) If $n=2, F_{0}$ and $F_{1}$ are independent along the reference trajectory. If $n \geq 3, F_{0}$ does not belong to 
$\operatorname{Span}\left\{\operatorname{ad}^{k} F_{0} \cdot F_{1}(x(t)) \mid k=0, \ldots, n-3\right\}$ along the reference trajectory.

The extremal is either exceptional and contained in the level set $\{H=0\}$, or normal. In the latter case, the classification is done according to the definition hereafter.

Definition 3.1: A normal extremal is said to be hyperbolic if $\left\{H_{1},\left\{H_{0}, H_{1}\right\}\right\}<0$, elliptic if $\left\{H_{1},\left\{H_{0}, H_{1}\right\}\right\}>0$.

We recall the following result from [2].

Theorem 3.1: Under our assumptions, let $(x, p, u)$ be the reference singular extremal defined on $[0, T]$. In the exceptional and hyperbolic (resp. elliptic) case, it is locally time minimizing (resp. maximizing) with respect to all trajectories with same extremities in the $\mathscr{C}^{0}$-topology up to the first conjugate time $t_{1 c}$. It is not locally time minimizing in the $\mathrm{L}^{\infty}$-topology after $t_{1 c}$.

Let us now define conjugate times, in the normal case first. If the extremal is hyperbolic (or elliptic), let us define

$$
\begin{aligned}
X= & \left\{p_{0} \in \mathbf{S}^{n-1} \subset T_{x_{0}}^{*} M \mid H_{1}\left(x_{0}, p_{0}\right)=\right. \\
& \left.\left\{H_{0}, H_{1}\right\}\left(x_{0}, p_{0}\right)=0\right\}
\end{aligned}
$$

and let $L_{0}=\mathbf{R} F_{1}\left(x_{0}\right) \oplus T_{p_{0}} X$, which is of dimension $n-2$. Then, a conjugate time $t_{c}$ is defined as a point such that

$$
\operatorname{rank}\left\{d \Pi\left(z\left(t_{c}\right)\right) \cdot L_{t_{c}}, F_{1}\left(x\left(t_{c}\right)\right)\right\}<n-1 .
$$

Analogously, in the exceptional case, let

$$
\begin{aligned}
X=\left\{p_{0} \in \mathbf{S}^{n-1}\right. & \subset T_{x_{0}}^{*} M \mid H_{0}\left(x_{0}, p_{0}\right)= \\
& \left.H_{1}\left(x_{0}, p_{0}\right)=\left\{H_{0}, H_{1}\right\}\left(x_{0}, p_{0}\right)=0\right\}
\end{aligned}
$$

and let $L_{0}=\mathbf{R} F_{1}\left(x_{0}\right) \oplus T_{p_{0}} X$, which is now of dimension $n-3$. A conjugate time $t_{c}$ is defined as a point where

$$
\operatorname{rank}\left\{d \Pi\left(z\left(t_{c}\right)\right) \cdot L_{t_{c}}, F_{1}\left(x\left(t_{c}\right)\right), F_{0}\left(x\left(t_{c}\right)\right)\right\}<n-1 .
$$

\section{KEPLER EQUATION FOR ORBIT TRANSFER}

We consider the minimum time control of the Kepler equation

$$
\ddot{q}=-q \frac{\mu}{r^{3}}+\frac{F}{m}
$$

where $q$ is the position of the satellite measured in a fixed frame $I, J, K$ whose origin is the Earth center, $r=|q|$, and $\mu$ the gravitation constant. The free motion where $F=0$ is the Kepler equation. The thrust is bounded, $|F| \leq F_{\max }$, and the mass variation is described by

$$
\dot{m}=-\beta|F|
$$

where $\beta$ is a positive constant. Written in the 3D radialorthoradial frame, the dynamics becomes

$$
\dot{x}=F_{0}+\frac{1}{m}\left(u_{r} F_{r}+u_{\text {or }} F_{\text {or }}+u_{c} F_{c}\right)
$$

with

$$
\begin{aligned}
& F_{r}=\frac{q}{|q|} \frac{\partial}{\partial \dot{q}} \\
& F_{c}=\frac{q \wedge \dot{q}}{|q \wedge \dot{q}|} \frac{\partial}{\partial \dot{q}}
\end{aligned}
$$

TABLE I

BOUNDARY CONDITIONS.

\begin{tabular}{lll}
\hline Variable & Initial cond. & Final cond. \\
\hline$P$ & $11.625 \mathrm{Mm}$ & $42.165 \mathrm{Mm}$ \\
$e_{x}$ & 0.75 & 0 \\
$e_{y}$ & 0 & 0 \\
$h_{x}$ & 0.0612 & 0 \\
$h_{y}$ & 0 & 0 \\
$l$ & $\pi \mathrm{rad}$ & $103 \mathrm{rad}$ \\
\hline
\end{tabular}

TABLE II

PHYSICAL CONSTANTS.

\begin{tabular}{ll}
\hline Variable & Value \\
\hline$\mu$ & $5165.8620912 \mathrm{Mm}^{3} \cdot \mathrm{h}^{-2}$ \\
$\beta$ & $1.42 e-2 \mathrm{Mm}^{-1} \cdot \mathrm{h}$ \\
$m^{0}$ & $1500 \mathrm{~kg}$ \\
$F_{\max }$ & $3 \mathrm{~N}$ \\
\hline
\end{tabular}

and $F_{\text {or }}=F_{c} \wedge F_{r}$. The boundary conditions define the initial and the terminal orbits, as well as positions on these. See Table I where they are given in terms of equinoctial elements [6].

Conjugate points for this problem can be computed by the algorithm of $\S$ II.

Indeed, any optimal control is smooth outside isolated points called $\Pi$-singularities where an instantaneous rotation of angle $p i$ occurs [6]. The norm of the control is thus (almost everywhere) maximum and the equation of the mass is solved by $m(t)=m_{0}-\beta F_{\max } t$. As a result, though non-autonomous, the system is a particular case of a $s u b$ Riemannian system for which the previous algorithm holds. Indeed, any smooth optimal control defines a singularity of the endpoint mapping where controls are taken on the sphere of radius $F_{\text {max }}$ : Although the system is affine in the command, controls can easily be reparameterized in order that the Legendre-Clebsh condition be satisfied. Test (6) is used in the normal case with free final time, and the rank is tested by a singular value decomposition of the $n-1=5$ Jacobi fields computed by cotcot. An equivalent test is to look for zeros of the determinant of the projection of Jacobi fields with the dynamics along the trajectory:

$$
\operatorname{det}\left(d \Pi\left(z\left(t_{c}\right)\right) \cdot L_{t_{c}}, \dot{x}\left(t_{c}\right)\right)=0 .
$$

The physical constants for numerical computation are summarized in Table II, and the result of the computation is shown in Fig. 1.

We end the section with the single-input case where the thrust is oriented along $F_{\text {or }}$. In the exceptional case, the control is the singular feedback $u_{e}=-D_{0} / D_{1}$ with

$$
\begin{aligned}
D_{0} & =F_{\text {or }}^{\prime} \wedge\left[F_{0}, F_{\text {or }}^{\prime}\right] \wedge\left[F_{0},\left[F_{0}, F_{\text {or }}^{\prime}\right]\right] \wedge F_{0} \\
& =2(v \wedge q)\left(|v|^{2}+\frac{\mu}{r}\right) \\
D_{1} & =F_{\text {or }}^{\prime} \wedge\left[F_{0}, F_{\text {or }}^{\prime}\right] \wedge\left[F_{\text {or }},\left[F_{0}, F_{\text {or }}^{\prime}\right]\right] \wedge F_{0} \\
& =-2 r^{2} q \cdot v .
\end{aligned}
$$

Since the system is of dimension 4 , according to $\S$ III we only have one Jacobi field to compute. The physical values 

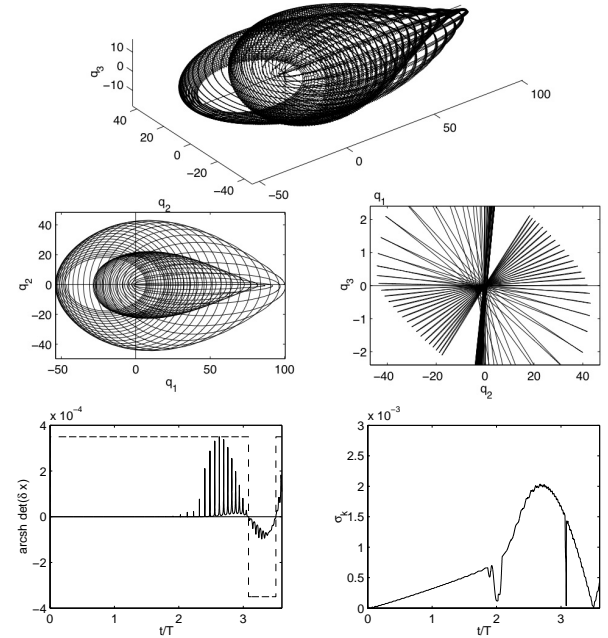

Fig. 1. A 3 Newton transfer. The minimum time is about 12 days, corresponding to 15 revolutions around the Earth, approximatively. On the top, the optimal trajectory (with projections in the equatorial plane and a perpendicular plane to illustrate how the inclination is corrected) is extended until roughly 3.5 times the minimum time. Bottom left, the determinant, bottom right, the smallest singular value of the Jacobi fields associated to the extremal. Two conjugate times are detected and the optimality is lost about 3 times the minimum time.
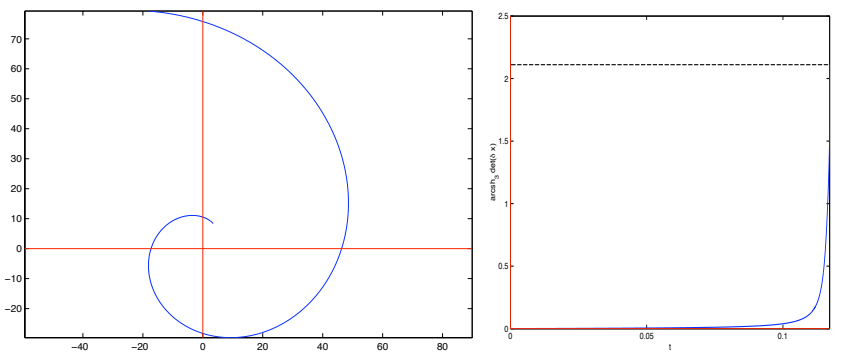

Fig. 2. An exceptional trajectory. The initial cumulated longitude $l_{0}$ is in ] $0, \pi\left[, l_{0}=3 \pi / 8\right.$, and the satellite spirals up so that $|q| \rightarrow \infty$, leaving rapidly the elliptic domain. The associated determinant remains negative, ensuring $\mathscr{C}^{0}$-local optimality of the whole trajectory.

for the computation are those of Table II. We set $u_{c}$ and $\beta$ to zero to have a $2 \mathrm{D}$-constant mass model, and we change the initial longitude not to start from the pericenter (see Fig. 2).

\section{EULER EQUATION FOR ATTITUDE CONTROL}

Recall that Euler equations are

$$
\begin{aligned}
& \dot{\Omega}_{1}=a_{1} \Omega_{2} \Omega_{3}+b_{1} u \\
& \dot{\Omega}_{2}=a_{2} \Omega_{1} \Omega_{3}+b_{2} u \\
& \dot{\Omega}_{3}=a_{3} \Omega_{1} \Omega_{2}+b_{3} u
\end{aligned}
$$

where

$$
a_{1}=\frac{I_{2}-I_{3}}{I_{1}}, a_{2}=\frac{I_{3}-I_{1}}{I_{2}}, a_{3}=\frac{I_{1}-I_{2}}{I_{3}} .
$$

We apply the intrinsic algorithm of computation of conjugate times presented in $\S$ III with the data $I_{1}=3, I_{2}=2, I_{3}=$ $1, b_{1}=2, b_{2}=1, b_{3}=1$. The test is achieved with

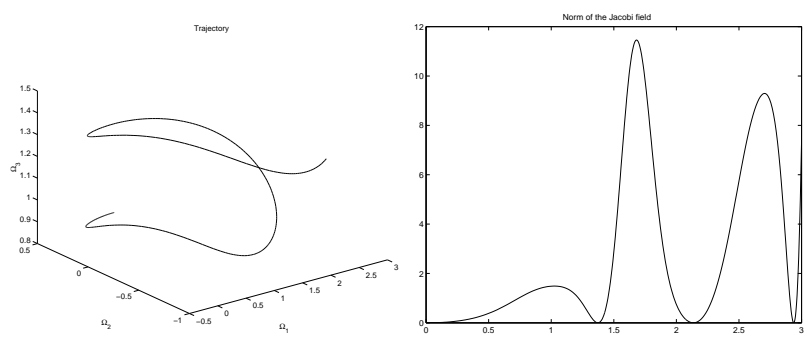

Fig. 3. Numerical results for Euler equations.

the initial values $\Omega_{1}=0.05, \Omega_{2}=0.05, \Omega_{3}=1$. The associated trajectory is hyperbolic, and we get a first conjugate time $t_{1 c} \simeq 1.37$, that corresponds to the vanishing of the norm of the unique Jacobi field computed by cotcot (see Fig. 3).

The complete system of attitude control of a rigid body consists in adding to Euler system the equations

$$
\dot{R}=S(\Omega) R
$$

where

$$
S(\Omega)=\left[\begin{array}{ccc}
0 & \Omega_{3} & -\Omega_{2} \\
-\Omega_{3} & 0 & \Omega_{1} \\
\Omega_{2} & -\Omega_{1} & 0
\end{array}\right] .
$$

The matrix $R(t)$ is a rotation matrix in $\mathbf{R}^{3}$, represented by an element of $\mathbf{R}^{9}$. To compute conjugate times, an alternative to $\S \mathrm{III}$ algorithm is to perform an integral transformation, namely the Goh transformation (see [2]), in order that the reduced system is regular in the sense of $\S I I$. The vector field $f_{1}$ being constant, one just has to change coordinates linearly. More precisely, assuming $b_{3} \neq 0$, we achieve the integral transformation by considering as a new control $v=x_{3}$, and we define the new coordinates

$$
x=\Omega_{1}-\frac{b_{1}}{b_{3}} \Omega_{3}, y=\Omega_{2}-\frac{b_{2}}{b_{3}} \Omega_{3} .
$$

The reduced system has the form

$$
\begin{aligned}
\dot{R} & =S(x, y, v) R \\
\dot{x} & =f_{1}(x, y, v) \\
\dot{y} & =f_{2}(x, y, v)
\end{aligned}
$$

where $f_{1}$ and $f_{2}$ are quadratic. For the numerical simulations, the initial data on the state (that is an element of $\mathbf{R}^{11}$ ) are $R(0)=I, x(0)=0.05, y(0)=0.05$. If we choose the initial adjoint vector $p_{0}=\left(\begin{array}{l}1 \\ 11111111111\end{array}\right)$ we are in the hyperbolic case. Observe that, except at a conjugate time, the rank is equal to 4 . Figure 4 represents the second, third and last singular values, and the first conjugate time corresponds to the vanishing of the fourth one. We get $t_{1 c} \simeq 285.729$.

If we choose the initial adjoint vector $p_{0}$ as before but change the third component according to $p_{0,3}=$ 0.99355412876393 , we are in the exceptional case of $\S$ III. Observe that, except at a conjugate time, the rank is equal to 3 . Figure 5 represents the second and third singular values, and the first conjugate time corresponds to the vanishing of the third one. We get $t_{1 c} \simeq 108.1318$. 

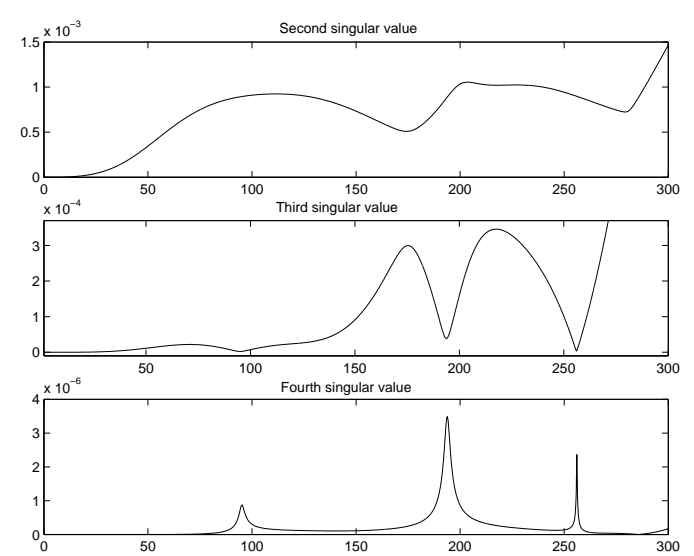

Fig. 4. Numerical results on attitude control, hyperbolic case.

\section{REFERENCES}

[1] A. A. Agrachev and A. V. Sarychev, "On abnormal extremals for Lagrange variational problems," J. Math. Syst. Estim. Cont., vol. 8, no. 1, pp. 87-118, 1998.

[2] B. Bonnard and I. Kupka, "Théorie des singularités de l'application entrée-sortie et optimalité des trajectoires singulières dans le problème du temps minimal," Forum Mathematicum, vol. 5, pp. 111-159, 1993.

[3] A. V. Sarychev, "The index of second variation of a control system," Math USSR Sbornik, vol. 41, pp. 338-401, 1982.

[4] B. Bonnard, J.-B. Caillau, and E. Trélat, "Cotcot: short reference manual," ENSEEIHT-IRIT, Technical report RT/APO/05/1, 2005.
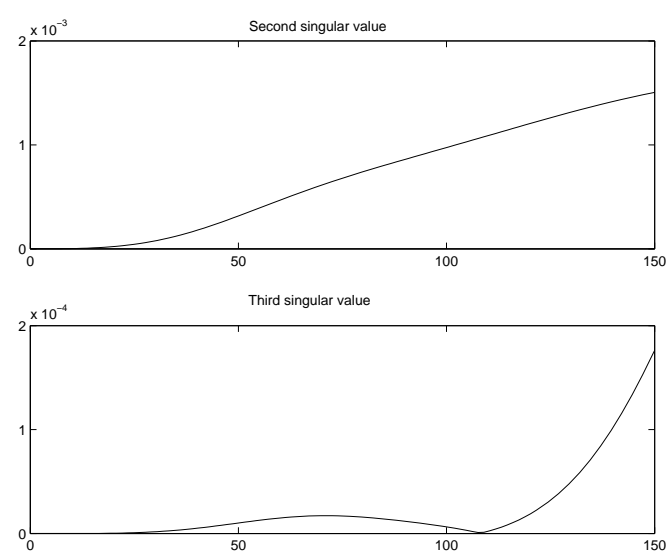

Fig. 5. Numerical results on attitude control, exceptional case.

[5] C. Bischof, A. Carle, P. Kladem, and A. Mauer, "Adifor 2.0: Automatic Differentiation of Fortran 77 Programs," IEEE Computational Science and Engineering, vol. 3, no. 3, pp. 18-32, 1996.

[6] B. Bonnard, J.-B. Caillau, and E. Trélat, "Geometric optimal control of Keplerian orbits," Discrete Cont. Dyn. Syst. Series B, vol. 5, pp. 929-956, 2005.

[7] — " "Second order optimality conditions in the smooth case and applications in optimal control," Laboratoire d'analyse numérique et EDP, Paris-Sud University, Technical report 2004-41, 2005.

[8] J. de Morant, "Contrôle en temps minimal des réacteurs chimiques discontinus," Ph.D. dissertation, Université de Rouen, 1992. 